
HERE TO EDIT) $<$

\title{
Analog radio-over-fiber transceivers based on III-V-on-silicon photonics
}

\author{
K. Van Gasse, L. Bogaert, L. Breyne, J. Van Kerrebrouck, S. Dhoore, C. Op de Beeck, A. Katumba, C.-Y. Wu, H. Li, \\ J. Verbist, A. Rahim, A. Abbasi, B. Moeneclaey, Z. Wang, H. Chen, J. Van Campenhout, X. Yin, B. Kuyken, G.
}

Morthier, J. Bauwelinck, G. Torfs, G. Roelkens

\begin{abstract}
Analog radio-over-fiber transceivers allow a substantial reduction in the complexity of the remote radio heads in the wireless network of the future. In this paper we discuss the building blocks for such a transceiver implemented on a silicon photonics platform, with the heterogeneous integration of III-V devices and the co-integration with electronics. Transmission experiments that demonstrate the viability of such integrated analog transceivers are described.
\end{abstract}

Index Terms- Radio-over-fiber, Silicon photonics, heterogeneous integration.

\section{INTRODUCTION}

$\mathrm{T}$ he 5th generation wireless network (5G), delivering gigabit-per-second connectivity to a massive number of users/devices with low latency, will require rethinking the wireless access network architecture as well as the hardware used. Meeting these ambitious targets will require substantially shrinking of the cell size of the network, thereby increasing the number of remote radio heads (RRHs). Cloud radio access networks (CRANs), where all the base station functions are centralized such that they can be efficiently shared over different antenna sites, are considered a very attractive approach to realize this. Besides the economic advantage of centralization, this approach also enables co-operative radio technologies to reduce the interference between the different RRHs, thereby allowing a further increase in their density. However, for the $5 \mathrm{G}$ network to be economically feasible, the complexity of the RRHs should be reduced. This requires rethinking the way the fronthaul fiber-optic communication between the central office and the antenna sites is realized. The options that are being considered are shown in Fig. 1. These include digital Radio-over-Fiber (DRoF), Sigma-Delta-overfiber (SDoF) and analog Radio-over-Fiber (ARoF). It is clear that, while DRoF can leverage the use of high-speed digital optical transceivers and the associated immunity to nonlinearities, they require the most complicated RRHs, as every RRH requires a de-serializer, digital-to-analog converter, mixer and oscillator to generate the high carrier frequency wireless signals. An interesting approach to simplify the RRH is to transmit a sigma delta modulated signal over fiber (SDoF). A sigma delta modulator oversamples the baseband signal and quantizes it to a 1-bit signal. A digital upconverter moves the signal to the required center frequency, after which the signal is

The authors acknowledge the UGent special research fund BOF, the European Research Council under Grant 695495 (ATTO), the H2020 5GPPP 5G-PHOS research program (ref.761989) for their financial support. Some of the high-speed equipment used in the work has been financed by the Hercules program of the Flemish government. K. Van Gasse, L. Bogaert, L. Breyne, S. Dhoore, C. Op de Beeck, A. Katumba, J. Verbist, A. Rahim, A. Abbasi, Z. Wang, B. Kuyken, G. Morthier and G. Roelkens are with the Photonics transmitted over fiber using the same digital optical transceivers as used in DRoF, making the transmission again immune to nonlinearities. At the antenna site only a bandpass filter is required to generate the RF signal. This approach was recently demonstrated in [1] for the transmission of $4 \times 3.5 \mathrm{Gbps} 256-$ QAM signals on a $3.5 \mathrm{GHz}$ carrier over $20 \mathrm{~km}$ of single mode fiber at $1310 \mathrm{~nm}$ and is a very attractive approach for sub-6 $\mathrm{GHz}$, lower baudrate communication systems. Moving to the millimeter wave frequency bands $(28 \mathrm{GHz}$ and $60 \mathrm{GHz})$ and exploiting the wide bandwidth available in these frequency bands, will likely require moving to ARoF links, where a millimeter-wave signal, generated in the central office, is modulating an optical carrier. This results in the simplest RRH configuration as it only requires the optical-to-electrical $(\mathrm{O} / \mathrm{E})$ conversion and electrical amplification. However, the main drawback of this approach is its sensitivity to nonlinearities in the $\mathrm{E} / \mathrm{O}$ and $\mathrm{O} / \mathrm{E}$ conversions and to transmission impairments such as dispersion. In this paper we will focus on the implementation of ARoF transceivers implemented on a III-Von-silicon photonic integrated circuit platform. The use of such a platform enables low cost transceiver manufacturing in high volume, very much needed for the wireless communication network of the future. We will focus on the different photonic components required to realize such links. On the transmit side these include III-V-on-silicon directly modulated and externally modulated lasers. For very high carrier frequencies $(60 \mathrm{GHz})$ we also describe the concept of photonic upconversion to reduce the bandwidth requirements on the opto-electronic components.

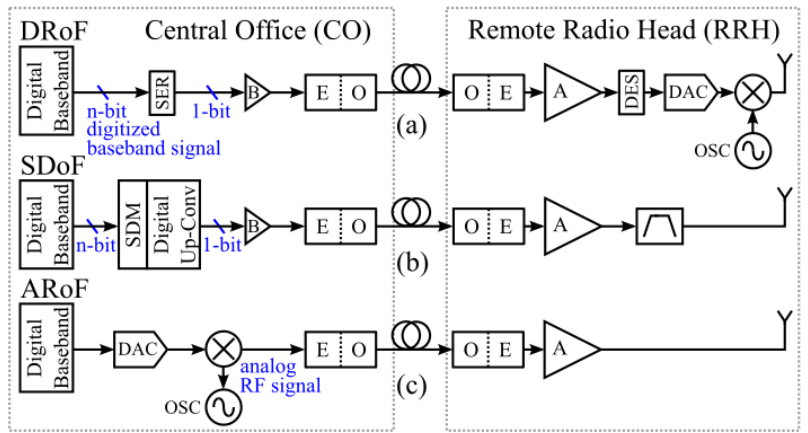

Fig. 1. Potential implementations of radio-over-fiber: (a) Digital Radio-overFiber (DRoF); (b) Sigma-Delta Radio-over-Fiber (SDoF); (c) Analog Radioover-Fiber (ARoF).

Research Group, Department of Information Technology, imec and Center for Nano- and Biophotonics, Ghent University, Ghent B-9000, Belgium (e-mail: Gunther.Roelkens@UGent.be). Joris Van Campenhout is with imec, Leuven (e-mail: Joris.Vancampenhout@imec.be). L. Bogaert, L. Breyne, J. Van Kerrebrouck, C.-Y. Wu, H. Li, J. Verbist, B. Moeneclaey, X. Yin, J. Bauwelinck and G. Torfs are with IDLab, Ghent University - imec, Ghent, Belgium (e-mail: Guy.torfs@UGent.be). 
On the receiver side we will describe the use of Ge (avalanche) photodetectors co-integrated with electrical amplifiers and traveling wave photodetectors with high saturation power, allowing to shift the amplification to the optical domain. Finally, we will demonstrate the potential of this integration platform through a number of system demonstrations.

\section{INTEGRATED AROF TRANSMITTER COMPONENTS}

Depending on the carrier frequency and complexity of the modulation format different transmitter architectures can be used. When realized on a silicon photonics platform these transmitters always require the integration of III-V semiconductors. This can be realized through flip-chip integration, die-to-wafer bonding, transfer printing or heteroepitaxial growth [2]. The devices reported in this paper are realized through die-to-wafer bonding. To reduce the cost full duplex links are considered, with the downstream in the C-band and the upstream in the O-band. In this paper we focus on the C-band downstream link. The simplest transmitter approach consists of using a directly modulated III-V-on-silicon distributed feedback laser (DML), as shown in Fig. 2(a) [3]. However, the typically limited bandwidth of these devices restricts the carrier frequency. Nevertheless, using a device with a relaxation oscillation frequency of $10 \mathrm{GHz}$ we were able to demonstrate 64-QAM OFDM transmission at $3.5 \mathrm{GHz}$ and 5 $\mathrm{GHz}$ carrier frequency and even 16 Gbps 16-QAM data transmission at $20 \mathrm{GHz}$ carrier frequency [4]. The transmission of complex modulation formats demonstrates also that the linearity of a directly modulated transmitter is sufficient for this purpose. For higher carrier frequency signals one needs to rely on external modulation, either by implementing a III-V-onsilicon transmitter with III-V electro-absorption modulator (EAM) (see Fig. 2(b)) [3] or using a silicon Mach-Zehnder intensity modulator (MZM) or IQ-modulator. EAM-based devices suffer from the transmission-versus-voltage nonlinearity of the device, therefore we will focus here on external silicon modulators.
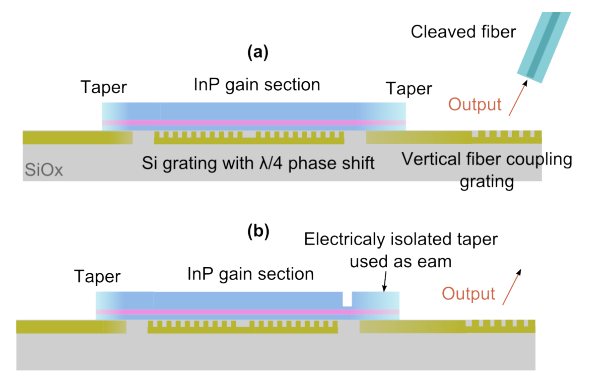

(c)

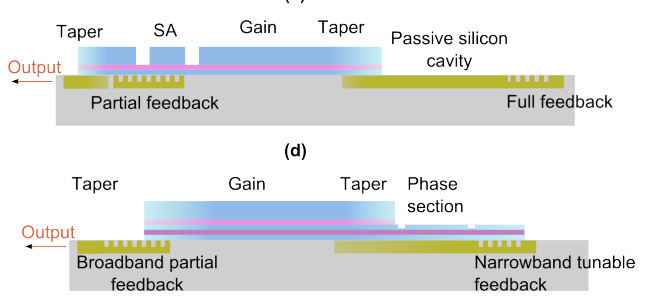

Fig. 2. III-V-on-silicon laser architectures: (a) distributed feedback laser; (b) externally modulated lasers using an EAM; (c) mode-locked laser; (d) distributed Bragg reflector laser.
While standard traveling wave silicon MZMs with high bandwidth and reasonable $\alpha \mathrm{V}_{\mathrm{pi}} \mathrm{L}_{\mathrm{pi}}$ figure of merit can be obtained [5], the narrow band nature of a radio-over-fiber signal allows exploring other modulator designs that provide a resonant enhancement of the RF signal applied to the modulator. One such approach is shown in Fig. 3(a) where a resonant electrode structure is used to enhance the modulation efficiency in a frequency band of interest (in this case the 20 $\mathrm{GHz}$ frequency band). IQ-modulators on the other hand are interesting to generate single-sideband modulated signals (which don't suffer from fading due to dispersion over the optical fiber) or carrier-suppressed single sideband (CS-SSB) signals for photonic upconversion. A push-pull silicon IQ modulator with $30 \mathrm{GHz}$ bandwidth is shown in Fig. 3(b). These modulators are implemented in imec's silicon photonics platform and are realized on $200 \mathrm{~mm}$ SOI wafers.
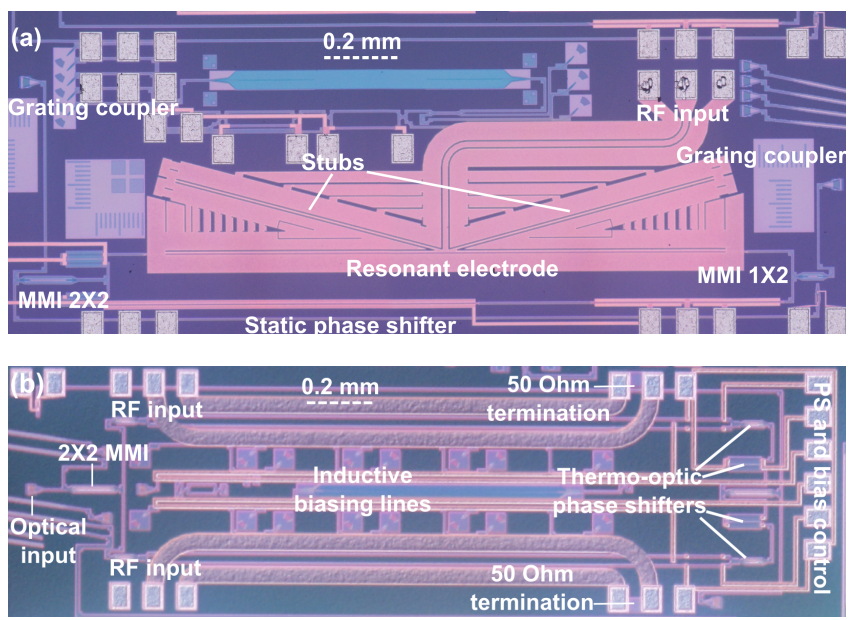

Fig. 3. (a) resonantly enhanced silicon Mach-Zehnder modulator; (b) silicon push-pull IQ modulator design

The concept of photonic upconversion is illustrated in Fig. 4, where the beating between two optical signals (one $\mathrm{CW}$, one CS-SSB) allows the upconversion of a baseband signal to any $\mathrm{RF}$ or mm-wave frequency. Either two free-running lasers can be used for this purpose, or the frequency difference between both can be stabilized by locking the lines to a reference frequency comb, implemented as a mode-locked laser. The locking can be realized using an optical phase-locked loop (OPLL) [6].

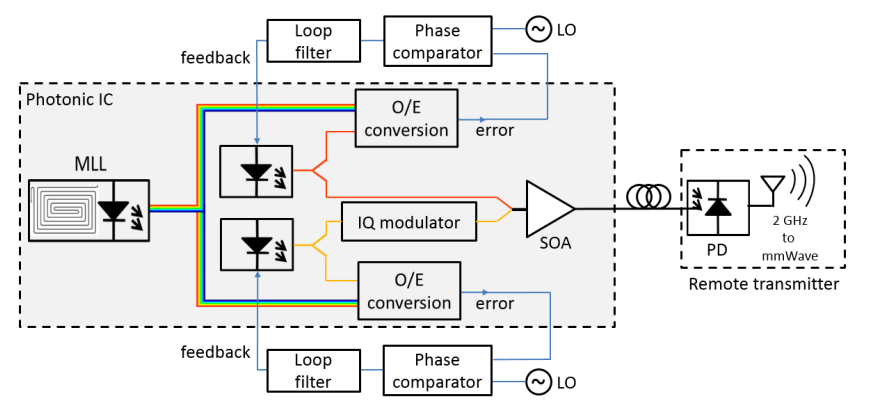

Fig. 4. Photonic upconversion concept with two $\mathrm{CW}$ tunable lasers locked to a mode locked laser frequency comb using an optical phase locked loop 


\section{HERE TO EDIT) <}

All these opto-electronic components can be integrated on a IIIV-on-silicon photonic integrated circuit. In Fig. 2(c) the longitudinal cross-section of a III-V-on-silicon anti-colliding pulse mode-locked laser is shown [7], while in Fig. 2(d) the longitudinal cross-section of a III-V-on-silicon distributed Bragg reflector (DBR) laser is shown [8]. Using such device geometries we have demonstrated mode-locked lasers with 1 $\mathrm{GHz}$ repetition rate and with a bandwidth of over $10 \mathrm{~nm}(>1$ $\mathrm{THz}$ ). The RF spectrum of the pulse train is shown in Fig. 5. The DBR lasers allow tuning over $>10 \mathrm{~nm}$ (microsecond-scale thermal tuning) and $>2 \mathrm{~nm}$ (nanosecond-scale electronic tuning). The 90 degree hybrids with integrated $\mathrm{Ge}$ photodetectors / transimpedance amplifiers needed for the OPLL can also be integrated in a straightforward way [9].
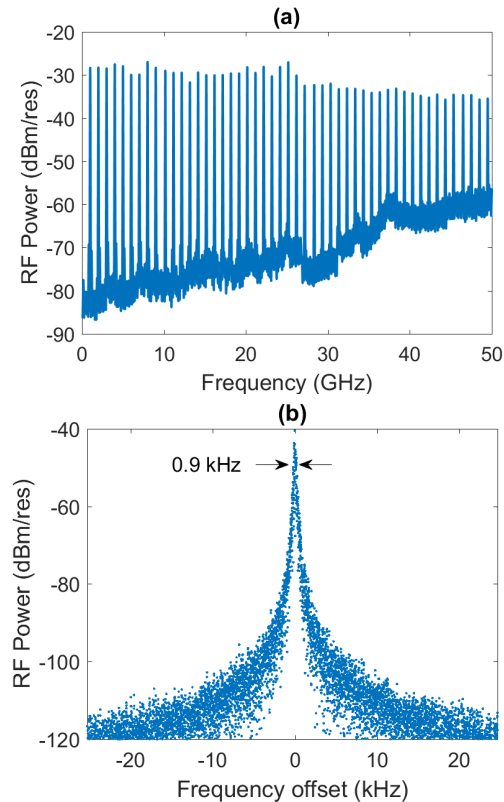

Fig. 5. III-V-on-silicon mode-locked laser RF spectrum (a) and zoom-in on the fundamental tone (b)

In order to boost the optical output power a III-V-on-silicon booster amplifier can be co-integrated with the transmitter. Recently we demonstrated III-V-on-silicon SOAs with a gain of $23 \mathrm{~dB}$ and a saturation power of $16 \mathrm{dBm}$.

\section{INTEGRATED AROF RECEIVER COMPONENTS}

On the receiver side the optical signal needs to be converted back to the electrical/wireless domain. This requires high speed photodetectors that are either co-integrated with a high-speed transimpedance amplifier [4] and antenna or consists of a photodetector directly connected to an antenna [10], in which case the optical signal needs to be pre-amplified. While the latter approach requires less components in the remote radio head, it requires high-speed photodetectors with high saturation current. For the classical electrical amplification scheme resonant transimpedance amplifiers (TIAs) making use of series inductive peaking are proposed for RoF communication links [11]. Such a band-pass approach benefits from superior noise behavior and a relaxation of the transimpedance limit compared to the traditional broadband designs typically used in RoF. Whereas the first property can also be obtained by combining the broadband TIA with a band-pass filter, the second cannot.
150 MBaud 64-QAM data reception on a $4 \mathrm{GHz}$ carrier frequency was demonstrated using such a receiver [11]. An alternative approach is to use a low noise amplifier (LNA) in between the photodetector and the antenna. To this end, a 3 stage 0.1 um pHEMT GaAs LNA was designed in the PH10 technology platform of UMS. The LNA is optimized for a Ge photodetector of the iSiPP silicon photonics platform from imec [5] and can be seen in Fig. 6. The design provides $24.6 \mathrm{~dB}$ gain from the Ge photodetector to a $50 \Omega$ load with a noise figure of only $2.04 \mathrm{~dB}$. Additionally, matching of at least $-12 \mathrm{~dB}$ at input and output is obtained over the entire 27.5 to $29.5 \mathrm{GHz}$ band. The LNA has an output referred $1 \mathrm{~dB}$ compression of $13.9 \mathrm{dBm}$.

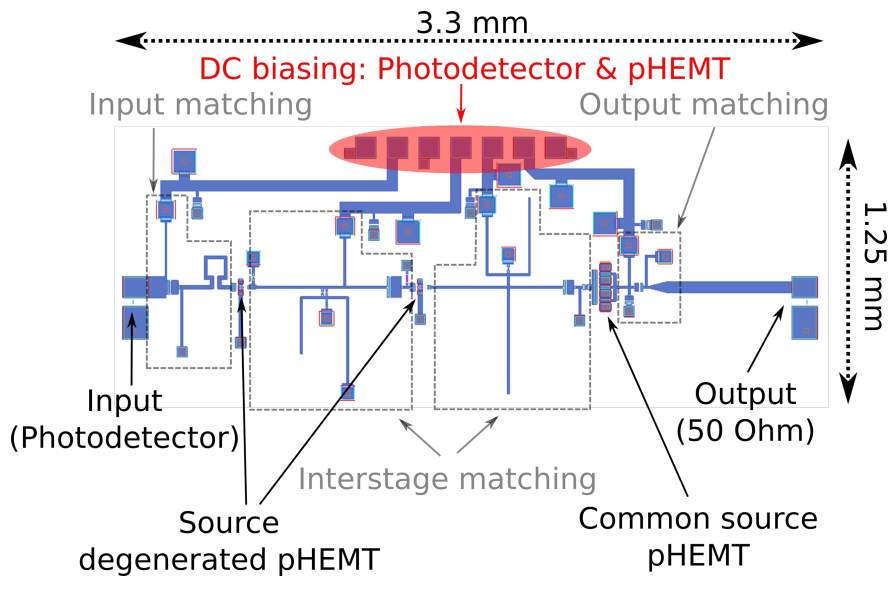

Fig. 6. $0.1 \mu \mathrm{m}$ GaAs LNA design for the $28.5 \mathrm{GHz}$ band

When the amplification is to be shifted from the electrical to the optical domain, a high-linearity, high-speed and high-powerhandling opto-electronic conversion is required. With a traveling wave photodetector the linearity can be increased while maintaining the high-speed properties of the standalone Ge photodetectors. Adopting a star coupler for the power distribution at the input [12] preserves the high linearity obtained from the traveling wave configuration. The traveling wave photodetector depicted in Fig. 7 (using 16 photodetectors) has a measured $3 \mathrm{~dB}$ bandwidth of $27.5 \mathrm{GHz}$ and has a fairly flat $\mathrm{S}_{21}$ up to $50 \mathrm{GHz}$ (less than $1 \mathrm{~dB}$ extra loss).

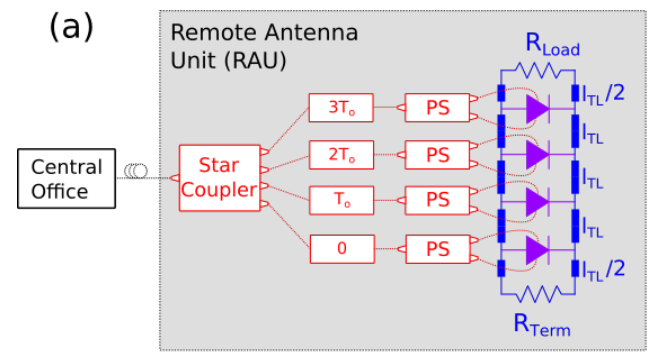

(b)

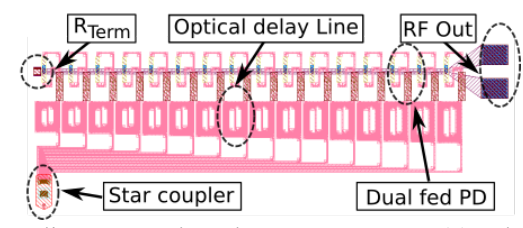

Fig. 7 Germanium traveling wave photodetector structure: (a) schematic; (b) gds of the fabricated device (PS: Power Splitter, To: Optical Delay, 1TL: transmission line segment length) 
Furthermore, the IP3 linearity at $28 \mathrm{GHz}$ is improved from $1.79 \mathrm{dBm}$ for a single Ge photodiode to $20.98 \mathrm{dBm}$ by adopting the dual-fed traveling wave design from Fig. 7.

\section{TRANSMISSION EXPERIMENTS}

To demonstrate the use of III-V-on-silicon photonic components in analog optical links several transmission experiments were performed [4]. An overview of the described links in shown in Fig. 8. For every transmission experiment a representative constellation diagram is shown. In a first experiment we investigated high data rate (16 Gbps) transmission on a $20 \mathrm{GHz}$ carrier using 16-QAM over $5 \mathrm{~km}$ of standard single mode fiber (SSMF) and achieved a BER lower than $10^{-5}$ (7\% rms EVM). In this experiment a III-V-on-silicon directly modulated laser was used in combination with a waveguide-coupled Ge photodiode coupled to a $0.13 \mu \mathrm{m}$ BiCMOS transimpedance amplifier. In a second experiment we transmitted a $120 \mathrm{Mbit} / \mathrm{s}$ LTE-type signal (64-QAM OFDM) on a 3.5 and $5 \mathrm{GHz}$ carrier over $5 \mathrm{~km}$ of SSMF using a similar configuration. An RMS error vector magnitude of $1.7 \%$ is achieved, attesting the good linearity of the DML and optical receiver. In a follow-up experiment this LTE transmission was combined with a 50 Gbps discrete multitone (DMT) transmission using the same transmitter and receiver [13]. Finally we also demonstrated a silicon photonic upconvertertransmitter, were two Ge-on-Si EAMs [5] are used in a MachZehnder configuration. One EAM is driven by a $120 \mathrm{Mbit} / \mathrm{s}$ 64QAM data on a $1.5 \mathrm{GHz}$ IF while the second EAM is driven with a $25 \mathrm{GHz} \mathrm{LO}$ signal. The result is a 64-QAM signal on a $26 \mathrm{GHz}$ carrier with a measured rms EVM of $3 \%$ at the receiver. The benefit of this approach is that the mixing happens in the optical domain and the mixer and transmitter are combined on one PIC.

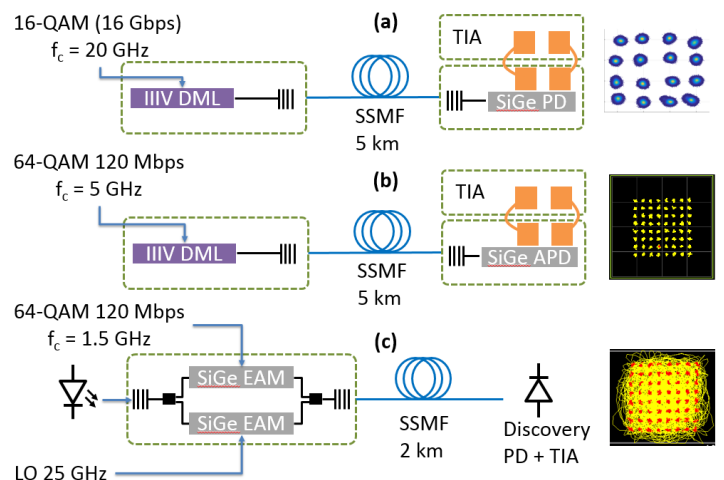

Fig. 8. Overview of analog transmission experiments with representative constellation diagrams. (a) 16-QAM signal on $20 \mathrm{GHz}$ carrier; (b) LTE-type signal on a $3.5-5 \mathrm{GHz}$ carrier; (c) $26 \mathrm{GHz}$ Ge-on-Si EAM photonic mixertransmitter.

\section{CONCLUSIONS}

III-V-on-silicon photonic integrated circuit technology is a promising platform to implement advanced analog optical transceivers. Different types of transmitters and receivers (cointegrated with the receiver electronics) have been demonstrated in this paper paving the way to low-cost transceivers for the wireless communication network of the future. While the transmitters and receivers have been demonstrated separately, their co-integration is still under development. Especially the integration of the III-V semiconductor devices on a full silicon photonics platform needs to be demonstrated. We believe that for future implementations transfer printing [14] is an enabling technology to realize this integration in a cost-effective way with high-throughput.

\section{REFERENCES}

[1] C.-Y. Wu. H. Li, J. Van Kerrebrouck, L. Breyne, L. Bogaert, P. Demeester, G. Torfs, "Real-time 4x3.5gbps Sigma Delta Radio-overFiber for a low-cost 5G C-RAN downlink," in Proc. Eur. Conf. Opt. Commun., Rome, Italy, 2018, Paper Tu3B.1.

[2] Z. Wang, A. Abbasi, U.D. Dave, A. De Groote, S. Kumari, B. Kunert, C. Merckling, M. Pantouvaki, Y. Shi, B. Tian, K. Van Gasse, J. Verbist, R. Wang, W. Xie, J. Zhang, Y. Zhu, J. Bauwelinck, X. Yin, Z. Hens, J. Van Campenhout, B. Kuyken, R. Baets, G. Morthier, D. Van Thourhout, G. Roelkens, "Novel light source integration approaches for silicon photonics", Laser \& Photonics Reviews, 11(4), p.1700063 (2017).

[3] A. Abbasi, B. Moeneclaey, J. Verbist, X. Y in, J. Bauwelinck, G.-H. Duan, G. Roelkens, G. Morthier, "Direct and electro-absorption modulation of a III-V-on-silicon DFB laser at 56 Gbps," IEEE Journal of Selected Topics in Quantum Electronics, p.1501307 (2017).

[4] K. Van Gasse, J. Van Kerrebrouck, A. Abbasi, J.Verbist, G. Torfs, B. Moeneclaey, G. Morthier, X. Yin, J. Bauwelinck, G. Roelkens, "III-V-onsilicon photonic transceivers for Radio-over-Fiber links," accepted for publication in J. Lightw. Technol. (2018).

[5] M. Pantouvaki, A. Srinivasan, Y. Ban, P. De Heyn, P. Verheyen, B. Snyder, M. Rakowski, J. De Coster, G. Lepage, H. Chen, N. Golshani, P. Absil, J. Van Campenhout, "Active Components for 50Gb/s NRZ-OOK Optical Interconnects in a Silicon Photonics Platform," IEEE J. Lightw. Technol. 35(4), p. 631-638 (2016).

[6] M. Lu, H. Park, E. Bloch, A. Sivananthan, A. Bhardwaj, Z. Griffith, L. A. Johansson, M. Rodwell, L. Coldren, "Highly integrated optical heterodyne phase-locked loop with phase/frequency detection," Optics Express 20(9), p. 9736-9741 (2012).

[7] Z. Wang, K. Van Gasse, Valentina Moskalenko, Sylwester Latkowski, Erwin Bente, B. Kuyken, G. Roelkens, "A III-V-on-Si ultra dense comb laser," Light: Science \& Applications, 6, p.e16260 (2017)

[8] S. Dhoore, G. Roelkens, G. Morthier, "III-V-on-silicon three-section DBR laser with over $12 \mathrm{~nm}$ continuous tuning range," Optics Letters, 42(6), p.1121-1124 (2017)

[9] J. Verbist, J. Zhang, B. Moeneclaey, W. Soenen, J. Van Weerdenburg, R. Van Uden, C. Okonkwo, J. Bauwelinck, G. Roelkens, X. Yin, "A 40 Gbaud QPSK/16-QAM integrated silicon coherent receiver," IEEE Photonics Technology Letters, 28(19), p.2070-2073 (2016)

[10] G. Torfs, H. Li, S. Agneessens, J. Bauwelinck, L. Breyne, O. Caytan, W. Joseph, et al. 2018. "ATTO: Wireless Networking at Fiber Speed." Journal of Lightwave Technology 36 (8): 1468-1477.

[11] L. Bogaert, J. Van Kerrebrouck, A. Abbasi, J. Lambrecht, G. Torfs, X. Yin, G. Roelkens, J. Bauwelinck, "Resonant optical receiver design by series inductive peaking for sub-6 GHz RoF," Microwave and Optical Technology Letters, 59(9), p.2279-2284 (2017)

[12] T. Spuesens, S. Pathak, M. Vanslembrouck, P. Dumon, W. Bogaerts, "Grating Coupler with Integrated Power Splitter for HighIntensity Optical Power Distribution," Photonics Technology Letters, 28(11), p.1173-1176 (2016)

[13] A. Rahim, A. Abbasi, M. Shahin, N. Sequeira Andre, A. Richter, J. Van Kerrebrouck, K. Van Gasse, A. Katumba, B. Moeneclaey, X. Yin, G. Morthier, R. Baets, G. Roelkens, "50 Gb/s DMT and $120 \mathrm{Mb} / \mathrm{s} \mathrm{LTE} \mathrm{signal}$ transmission over $5 \mathrm{~km}$ of optical fiber using a silicon photonics transceiver," Advanced Photonics Congress p. IW1B.4 (2018).

[14] J. Zhang, B. Haq, J. O'Callaghan, A. Gocalinska, E. Pelucchi, A. Jose Trindade, B. Corbett, G. Morthier, G. Roelkens, "Transfer-printing-based integration of a III-V-on-silicon distributed feedback laser," Optics Express, 26(7), United States, p.8821-8830 (2018) 